\title{
Protoporphyrie héréditaire en race bovine Limousine française, premiers résultats
}

\author{
JJ Lauvergne ${ }^{1}$, L Pinault ${ }^{2}$ \\ 1 Institut national de la recherche agronomique, laboratoire de génétique factorielle, \\ CRJ, 78352 Jouy-en-Josas; \\ 2 École nationale vétérinaire de Nantes, service de pharmacie et toxicologie CP 3013 , \\ 44087 Nantes cedex 03, France
}

(Reçu le 19 avril 1991; accepté le 6 mai 1991)

\begin{abstract}
Résumé - Un dosage par fluorimétrie de la teneur en porphyrines liées au zinc (PPZ) dans le sang de 112 taurillons Limousins d'un an nés en 1988 et testés pour la production de viande à la Station nationale de qualification de la race limousine de Lanaud près de Limoges a révélé l'existence de 2 animaux dont la teneur en PPZ $(\simeq 110 \mu \mathrm{g} / 100 \mathrm{ml})$ s'écartait de plus de 9 écarts types de la moyenne des autres animaux $(\bar{x}=24,5 \mu \mathrm{g} / 100$ $\mathrm{ml}$ ). Il pourrait s'agir de deux porteurs hétérozygotes de l'anomalie héréditaire récessive autosomique de protoporphyrie déjà décrite dans le rameau Limousin d'Amérique du Nord. Une estimation de la fréquence du gène dans le Limousin français serait alors $\widehat{q}=0,009$ avec $\sigma_{\widehat{q}}=0,006$. La probabilité d'apparition de veaux protoporphyriques serait alors d'environ 1 sur 13000 ( \pm 45 naissances anomaliques par an). Jointe à une insolation moins forte qu'en Amérique, cette faible probabilité d'apparition peut expliquer que les cas cliniques aient pu jusqu'à présent passer inaperçus en France alors que les premières observations américaines remontent à 1976. Des études complémentaires sont encore nécessaires.
\end{abstract}

protoporphyrie héréditaire / race bovine Limousine

Summary - Hereditary protoporphyria in French Limousin cattle : first results. $A$ fluorimetric measurement of blood content of zinc bound porphyrins (PPZ) on 112 1yr-old Limousin bulls born in 1988 and included in a performance test programme at the National Station for Qualification of the Limousin Breed of Lanaud near Limoges (France) has shown that for 2 of them the value $(\approx 110 \mu \mathrm{g} / 100 \mathrm{ml})$ was 9 standard deviations higher than the mean of the other $110: \bar{x}=24.5 \mu \mathrm{g} / 100 \mathrm{ml}$. These 2 bulls were considered to be heterozygotes carrying the autosomal recessive gene for protoporphyria already described in the American branch of the Limousin breed. An estimate of the gene frequency is $\widehat{q}=0.009$ with $\sigma_{\widehat{q}}=0.006$ which gives a probability for a protoporphyric birth of one in $13000: \approx$ 45 cases per year in the French Limousin. This low probability of occurrence added to the reduced isolation compared to America may explain why, up to now, no clinical cases of protoporphyria have been reported in France when the first description in America traces back to 1976. Complementary studies are still necessary.

hereditary protoporphyria / Limousin cattle 
La protoporphyrie est une affectation qui perturbe le métabolisme de la porphyrine dans le sang des mammifères (Nordmann, 1990). On en connaît au moins un cas, hérité mendéliennement en dominance autosomique à pénétrance incomplète chez l'homme (Mc Kusick, 1975, Deybach et al, 1986).

En 1976, l'examen d'une génisse Limousine souffrant de lésions cutanées a permis au Veterinary Diagnostic Laboratory de Brookings (Sud Dakota) de porter le premier diagnostic de ce type de maladie héréditaire dans l'espèce bovine (Ruth et al, 1977; Ruth et al, 1978).

La race Limousine qui a été importée de France au Canada puis aux Etats-unis à la fin des années 60 est devenue une race à viande très populaire dans les provinces occidentales du Canada et dans l'Ouest des Etats-Unis. Deux sociétés d'éleveurs la North American Limousin Foundation, (NALF, Denver, Colorado) aux EtatsUnis et la Canadian Limousin Association (CLA, Calgary, Alberta) au Canada regroupant respectivement 6000 et 3000 membres gèrent environ un million de reproducteurs.

Au début de 1989, l'un d'entre nous (JJL) fut alerté par un responsable de la NALF sur les conseils du Dr Leipold (Kansas State University) responsable du projet nord-américain de détection des anomalies héréditaires bovines. La NALF était désireuse de savoir si l'anomalie en question, bien que non encore décrite en France, ne pouvait pas néanmoins exister dans la race Limousine dont provenaient toutes les souches nord-américaines.

Des contacts furent alors pris avec l'UPRA (Unité de sélection et de promotion de race agréée) France Limousin sélection et le Service de pharmacie et toxicologie de l'Ecole nationale vétérinaire (ENV) de Nantes afin de procéder à un examen sanguin de bouvillons Limousins âgés d'un an environ en testage de performances bouchères à la Station nationale de qualification de la race Limousine de Lanaud près de Limoges.

La protoporphyrie décrite en race Limousine d'Amérique est une maladie qui provient d'une réduction de l'activité de l'enzyme appelée ferrochélatase impliquée dans le dernier stade de la formation de l'hémoglobine (Dailey et Fleming, 1983; Bloomert et al, 1987). Il en résulte une teneur élevée de prophyrines libres ( $\mathrm{P}$ libre) dans le sang, principalement la porphyrine IX, molécule qui sert de substrat à la ferrochélatase. Cet excès de P-libre en circulation déclenche des réactions de photosensibilité dans les zones dépourvues de poils et des lésions hépatiques (Schwartz et al, 1980).

Les indications données par les derniers auteurs cités laissaient penser que, chez les animaux présentant la tare héréditaire, une partie de la protoporphyrine non fixée au fer se trouvait complexée au zinc des hématies et entraînait la formation d'une quantité anormalement élevée du complexe protoporphyrine-zinc (PPZ) aisément détectable par fluorescence directe à l'aide d'un hématofluorimètre.

Ce taux de conversion reste cependant faible et non connu, contrairement à ce que l'on observe lors d'intoxication par le plomb ou lors de carences en fer.

Disposant d'un hématofluorimètre et aux fins de procéder à une étude exploratoire - à confirmer par d'autres mesures dont la mise au point puis la mise en œuvre sont plus lourdes - il a été décidé de procéder sans délais à un sondage sur un échantillon des futurs reproducteurs français de la race susceptibles de porter le gène d'anomalie en question. 
Les analyses effectuées sur le sang de 112 bouvillons âgés d'environ un an et prélevés les 18 et 19 mai 1989 à Lanaud ont consisté dans le dosage de la teneur en PPZ par hématofluorimétrie directe (hematofluorometer ESA Zn P 4000 ).

L'histogramme de distribution de ces concentrations en PPZ de la figure 1 montre que l'on est en présence d'une distribution normale ou sub-normale comprenant 110 valeurs entre 0 et $60 \mu \mathrm{g} / 100 \mathrm{ml}(n=110, \bar{x}=24,36, \sigma=10,9)$ et de 2 valeurs autour de $110 \mu \mathrm{g} / 100 \mathrm{ml}$ qui, manifestement, ne font pas partie de cette distribution (à près de 8 écarts types de la moyenne de la première distribution, la probabilité pour que la variable prenne une telle valeur est inférieure à 0,0001 ).

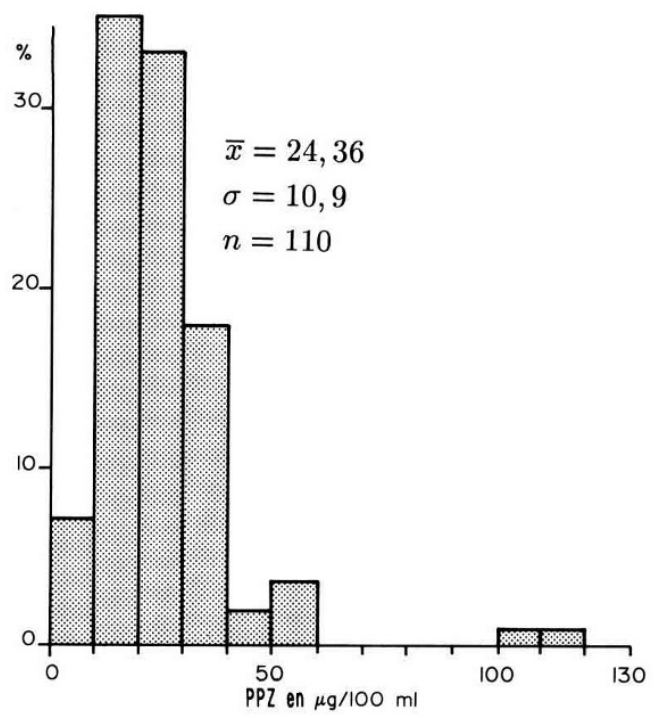

Fig 1. Histogramme des concentrations en porphyrines liées au zinc (PPZ), en $\mu \mathrm{g} / 100 \mathrm{ml}$, dans le sang de 112 bouvillons Limousins nés en 1988, âgés d'un an environ et prélevés à la Station Nationale de Qualification de Lanaud près de Limoges en mai 1989.

Nos données en elles-mêmes n'apportent aucune preuve directe de l'hérédité de l'anomalie puisque les 2 taurillons détectés par des teneurs anormales n'avaient aucun ancêtre commun sur 4 générations d'ascendants et qu'aucun dosage n'a été effectué chez les parents.

L'interprétation que l'on peut donner est que la première distribution regrouperait les homozygotes sains alors que les deux animaux dont la teneur est autour de $110 \mu \mathrm{g} / 100 \mathrm{ml}$ seraient des hétérozygotes porteurs sains du gène de l'anomalie. Cette interprétation procède par analogie avec les analyses des auteurs américains Ruth et al, (1977) qui distinguaient, d'après la teneur en protoporphyrines des érythrocytes, les homozygotes sains des hétérozygotes porteurs sains mais transmetteurs du gène et des homozygotes présentant les signes cliniques de la protoporphyrie.

Sous cette hypothèse et en considérant l'échantillon des 112 taurillons testées en 1989 à Lanaud comme un échantillon tiré au hasard dans la population Limousine 
française, on peut, pour estimer la fréquence $q$ du gène dans la race, utiliser l'équation du maximum de vraisemblance proposée par Elandt-Jonhson (1971) : $\widehat{q}=\left(r_{2}+2 r_{3}\right) / 2 n$ où $r_{2}$ est le nombre des hétérozygotes, $r_{3}$ celui des homozygotes malades et $n$ le nombre total des observations.

Il vient alors : $\sigma_{\widehat{q}}^{2}=q(1-q) / 2 n$

Introduisant nos données d'observations $r_{2}=2, r_{3}=0$ et $n=112$, on obtient : $\widehat{q}=0,0089 \simeq 0,9 \cdot 10^{-2}$ avec $\sigma_{\widehat{q}}=0,0063$

Il s'agit d'une fréquence génique faible qui donne seulement un nouveau né anomalique pour environ 13000 naissances $\left(\widehat{q}^{2}=0,8 \cdot 10^{-4}\right)$. Si l'on sait (Scees, 1990) qu'en 1988 il y avait en France 560000 vaches Limousines, on devrait enregistrer environ 45 naissances de veaux protoporphyriques par an.

Jointe au mode d'élevage des bovins Limousins en France - qui sont, d'une manière générale, soumis à une insolation moins intense qu'en Amérique - cette faible fréquence explique que l'anomalie ait pu, jusqu'à présent, passer inaperçue dans notre pays.

La fréquence dans le rameau américain n'est pas communiquée mais la lecture des articles de certains responsables (Vanderwert, 1988; Vanderwert, sd) laisse à penser qu'elle pourrait être plus élevée qu'en France, ce qui serait dû à une dérive génétique résultant d'un effet de fondateur.

Quoi qu'il en soit, on peut déjà avancer qu'un gène de protoporphyrie apparemment le même que celui qui a été décrit en Amérique - existe probablement dans la branche Limousine française, avec une fréquence de l'ordre de 1/100.

Des contrôles supplémentaires sont nécessaires pour préciser son mode de transmission héréditaire et estimer sa fréquence avec plus de précision.

\section{REMERCIEMENTS}

Les conseils du professeur HW Leipold (Kansas State University) ont été très précieux.

Les contacts avec le Président B Waddle et le Secrétaire Dr W Venderwert de la North American Limousin Foundation et le Secrétaire H Tedford de la Canadian Limousin Association ont été très enrichissants.

La collecte et l'expédition des échantillons a été organisée par l'UPRA France Limousin Sélection (Président L de Neuville, Directeur Général JN Bonnet) avec le concours de la Direction des Services Vétérinaires de la Haute-Vienne (M Sazerat).

Nous voudrions remercier également le Pr Y Nordmann, Directeur du Centre Français des Porphyries (Hôpital Louis Mourier, Colombes) qui a bien voulu nous faire profiter de son expérience et relire le manuscrit.

\section{RÉFÉRENCES}

Bloomert JR, Hill HD, Morton O, Anderson-Burnham LA, Straka JG (1987) The enzyme defect in bovine portoporphyria : studies with purified ferrochelatase. $J$ Biol Chem 262, 667-671

Dailet HA, Fleming E, (1983) Bovine ferrochelatase : kinetic analysis of inhibition by $N$-methylprotoporphyrin, manganese and heme. $J$ Biol Chem 258, 11453-11456 
Deybach JC, da Silva V, Pasquier Y, Nordmann Y (1986) Ferrochelatase in human erythropoietic protoporphyria : the first case of a homozygous form of the enzyme deficiency. In : Porphyrins and porphyrias. Colloque INSERM, John Libbey Eurotext Ltd, 136, 163-173

Eladt-Johnson RD (1971) Probability models and statistical methods in genetics. John Wiley and Cons, New York, 318-321

Mc Kusick V (1975) Mendelian inheritance in man. Catalog of autosomal dominant, autosomal recessive and $X$-linked phenotypes. The Johns Hopkins University Press, Baltimore, 278

Nordmann Y (1990) Porphyries héréditaires. Editions Techniques, Encycl Méd Chir Paris, France, Pédiatrie, $4059 \mathrm{Q}^{10}, 10-1990,6 \mathrm{p}$

Ruth GR, Schwartz S, Stephenson B (1977) Bovine protoporphyria : the first non human model of this hereditary photosensitizing disease. Science, 198, 199-201

Ruth GR, Schwartz S, Stephenson B, Bates F, Shave H (1978) A new disease in cattle, bovine protoporphyria, clinical and diagnostic features. $21^{\text {st }}$ Ann Proc Assn Vet Lab Diagnostic, 91-96

Scees (1990) Recensement Général Agricole, 1988. Ministère de l'Agriculture, Paris Schwartz S, Stephenson B, Sarkar D, Freyholtz H, Ruth G, (1980) Quantitative assay of erytrocyte "free" and zinc-protoporphyrin : clinical and genetic studies. Int J Biochem, 12, 1053-1057

Vanderwert W (1988) Protoporphyria awareness. Limousin World, Jan 1988, 47-49 Vanderwert W (sd) A visit to the Minnesota laboratory : more on Protoporphyria. North American Limousin Foundation, Denver (CO), mimeogr, $9 p$ 